\title{
LA PROBLEMÁTICA DE LA ARBOLEDA DEL "SOTO $Y$ PARTINCHAS" EN EL GALACHO DE JUSLIBOL (ZARAGOZA): DIAGNOSTICO Y ALTERNATIVAS DE ACTUACIÓN
}

\author{
Gustavo BARRON, Paloma IBARRA, Luis Alberto LONGARES, Fernando PEREZ y \\ Pedro SESE \\ Departamento de Geografía y Ordenación del Territorio \\ Universidad de Zaragoza
}

\begin{abstract}
Resumen: Se presenta la problemática de un espacio de ribera, la arboleda del "Soto y Partinchas", que contacta con el Espacio Natural Protegido del Galacho de Juslibol (Zaragoza). Se diagnostica su estado actual, sus funciones y la tendencia evolutiva prevista para, finalmente, exponer diferentes alternativas de actuación o gestión.
\end{abstract}

Palabras clave: Vegetación de ribera, Galacho de Juslibol, plagas, diagnóstico, evolución, gestión.

Abstract: A problematic of river banks space is presented, "Soto and Partinchas" woods, which contact the Protected Natural Space of the meander of Juslibol (Zaragoza). The real state, its functions and the foreseen evolutive tendency are diagnosed, so that, finally, present different options of acting or management.

Key words: River bank vegetation, meander of Juslibol, forest pests, diagnosis, evolution, management.

\section{INTRODUCCION Y PLANTEAMIENTO DEL PROBLEMA}

El denominado Soto y Partinchas se localiza en el sector más meridional del Galacho de Juslibol, meandro abandonado del río Ebro localizado en las proximidades de Zaragoza (Fig. 1). Hasta hace unos años constituía una arboleda bastante densa y bien estructurada compuesta por olmos (Ulmus minor) y chopos (Populus alba y 
Populus nigra) lo que justifica su consideración como una de las zonas de mejor conservación, mayor confort y mayor calidad ambiental dentro del conjunto del Galacho de Juslibol (Universidad de Zaragoza, 1990). Además, es una masa forestal que ha cumplido y cumple una importante función de "cierre" y protección de las zonas más frágiles del propio Galacho en su límite meridional. En el momento actual el soto ha experimentado una degradación y unos cambios importantes debido, principalmente, a que la enfermedad de la grafiosis ha afectado a la práctica totalidad de los olmos adultos de la arboleda causando su muerte.

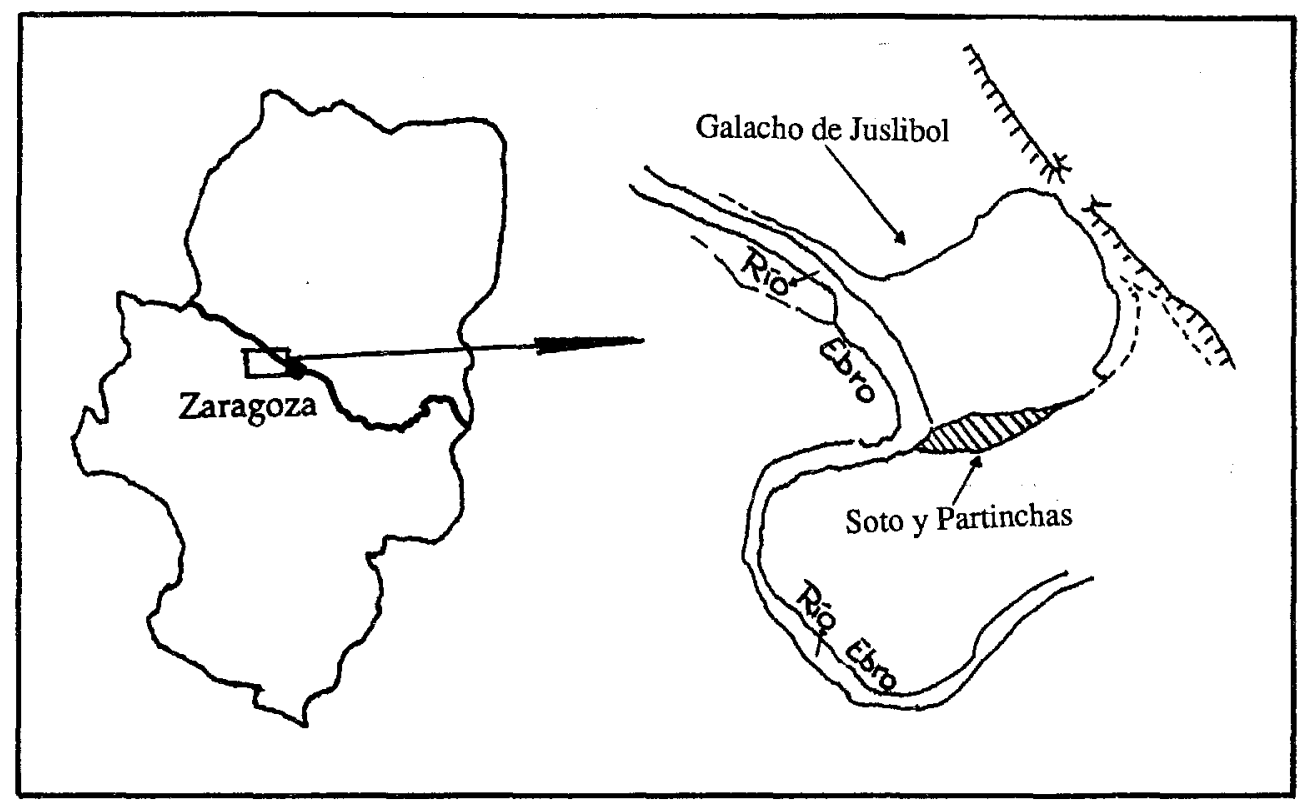

Figura 1.- Mapa de localización.

Por otra parte, el soto está incluido en el área de estudio del Programa MABUNESCO: el Hombre y la Biosfera, que tiene como uno de sus objetivos la recuperación y conservación de áreas naturales urbanas y sus aledaños. Estos objetivos entran en conflicto con el aprovechamiento economicista que los actuales propietarios quieren realizar del Soto y Partinchas. 
En consecuencia, los objetivos de este trabajo $^{1}$ son analizar el estado actual del soto y de sus áreas limítrofes, diagnosticar su tendencia evolutiva y, por último, analizar las diferentes alternativas de gestión, ya sean llevadas a cabo por los propietarios particulares o por la Administración.

\section{METODOLOGIA}

El proceso metodológico ha sido el siguiente:

- Recopilación de información bibliográfica, cartográfica y fotográfica de diferentes años.

- Análisis diacrónico a través de los diferentes vuelos aéreos disponibles, evidenciando los cambios experimentados tanto geomorfológicos como de vegetación.

- Trabajo de campo con reconocimiento del estado actual de los distintos tipos de formaciones vegetales diferenciadas en el soto.

- Entrevistas a técnicos municipales y a especialistas en temas fitosanitarios.

- Elaboración del diagnóstico sobre el estado actual y la tendencia evolutiva de la vegetación.

-Propuesta de tipos de actuaciones según diferentes criterios de gestión.

\section{EVOLUCION EN LAS ULTIMAS DECADAS Y SITUACION ACTUAL}

Para comprender los distintos tipos de formaciones vegetales que se diferencian en la actualidad en el Soto y Partinchas y sus áreas limítrofes así como su distribución espacial, es preciso hacer referencia a los importantes cambios experimentados en este sector en relación a la dinámica fluvial del Ebro.

En 1957 el Soto y Partinchas se corresponde con una formación vegetal densa, arbórea, compuesta fundamentalmente de olmos y chopos y con unos límites romboidales claramente marcados por el Ebro y los campos de cultivo (Fig. 2a); como

1 Este trabajo forma parte del "Estudio de Recuperación del Galacho de Juslibol. II parte", realizado para el Ayuntamiento de Zaragoza. 
puede observarse, todo el margen septentrional contacta directamente con la lámina de agua del Ebro.

Posteriormente, en 1961, tuvo lugar la corta que supuso un nuevo trazado del Ebro en este sector y la formación del propio Galacho o meandro abandonado (Fig. 2b). En consecuencia, la lámina de agua del Ebro únicamente "araña" ligeramente la superficie original del soto en su extremo SO., coincidiendo con la zona diagnosticada como de máxima fragilidad (Universidad de Zaragoza, 1990). A partir de ese momento, los procesos de regeneración vegetal se pusieron en marcha y provocaron progresivamente la conexión de la arboleda del Soto y Partinchas con la vegetación colonizadora del antiguo lecho y del interior del Galacho.

Las fotografías aéreas posteriores a 1961 (Figs. 2b y 2c), reflejan claramente estas transformaciones geomorfológicas e hidrológicas que trajeron como consecuencia grandes cambios en la vegetación del Galacho y de su interior y también en las áreas limítrofes al Soto y Partinchas más que en la vegetación de la propia arboleda o soto (al menos que puedan observarse en las fotografías aéreas a escalas medias). En efecto, en los fotogramas de 1974, 1981 y 1988, la cubierta vegetal del soto sigue apareciendo densa, homogénea y con una rugosidad y color característica de los bosques con buen recubrimiento de copas y ejemplares maduros. Sin embargo, aparecen nuevas formaciones vegetales en las áreas limítrofes inexistentes antes de la corta de 1961.

Así pues, en el momento actual, existen diversos tipos de comunidades vegetales en el entorno del Soto y Partinchas cuya presencia y distribución se explican por la variabilidad de los factores ambientales (humedad, suelo, salinidad, topografía, etc...), modificados en buena parte por la dinámica fluvial expuesta anteriormente $y$, en ocasiones, también por las intervenciones de tipo antrópico que han tenido lugar en este espacio.

El espacio del Soto y Partinchas propiamente dicho está formado por una olmeda-chopera y una chopera-olmeda, estando ambas formaciones delimitadas en su margen exterior por una orla espinosa. Esta orla exterior del soto contacta directamente con el dique construido por la Confederación Hidrográfica del Ebro que es utilizado como camino y que separa la arboleda de los campos de cultivo. Se trata una orla espinosa típica de límite de bosque, con dominio de los estratos arbustivo y arborescente y resultando densa y bastante impenetrable (sólo se puede acceder con comodidad al soto por algunos puntos concretos en los que se interrumpe esta orla). Son frecuentes los fresnos (Fraxinus angustifolia), los olmos jóvenes (Ulmus minor), los majuelos (Crataegus monogyna), las retamas (Retama sphaerocarpa) y sobre todo las zarzas (Rubus ulmifolius). 

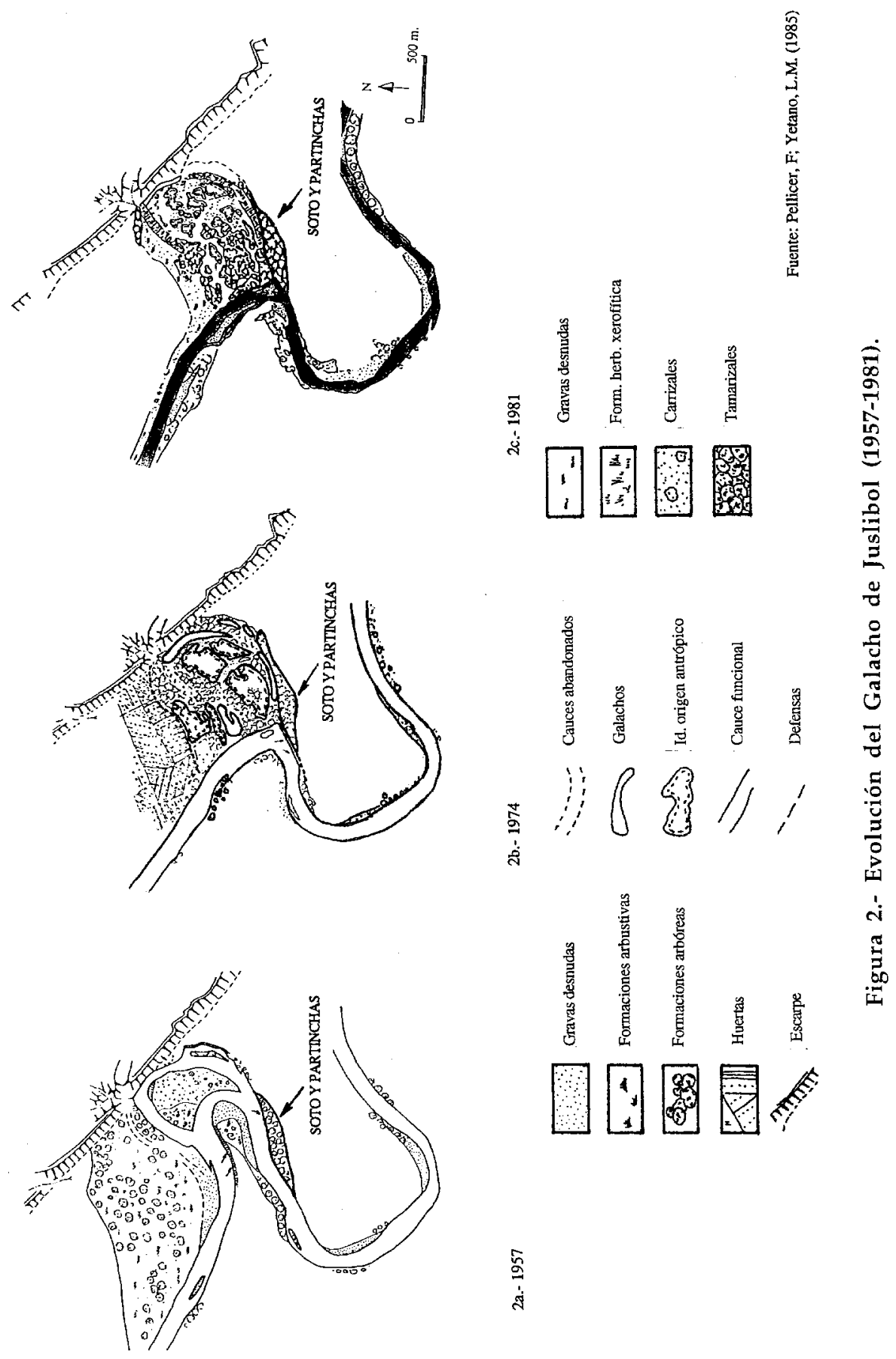
En el extremo occidental se puede individualizar un subsector en el que la orla descrita es prácticamente inexistente y en su lugar encontramos una banda estrecha casi monoespecífica de olmos muertos cuyos troncos aún permanecen en pié en un porcentaje importante.

La Olmeda-chopera es una formación arbórea mixta, compuesta fundamentalmente por Olmos (Ulmus minor) y por chopos (Populus nigra y Populus alba) en menor proporción; todos ellos son de elevado porte y el recubrimiento de copas era superior al $90 \%$ en los momentos previos a la grafiosis. Como se observa en la figura 3 , se localiza en el extremo más meridional y ocupa una superficie bastante reducida respecto al total del Soto y Partinchas. En el momento actual, los olmos han sido afectados de forma generalizada por plaga de la grafiosis. Por ello, al penetrar hoy en esta olmeda-chopera se tiene una impresión de desolación y abandono: lo que se encuentra es un amasijo de ramas y troncos secos de los olmos enfermos entrelazados en el suelo dificultando enormemente el tránsito, numerosos olmos muertos que todavía se mantienen en pie pero que pueden caer en cualquier momento (crujen movidos por el viento de forma lastimera $y$, ciertamente, peligrosa), rodales de zarzas y otras especies espinosas que han ido proliferando y hacen inaccesibles algunos espacios.

Son únicamente los chopos los que mantienen el estrato arbóreo de esta formación vegetal y dan la impresión de estar en un bosque, aunque esté muy degradado. En el sotobosque dominan las especies arbustivas, rosáceas (Rubus ulmifolius, Crataegus monogyna...) y otras espinosas, aunque también está presente un escaso estrato arborescente de sanguinos (Cornus sanguinea), chopos e incluso algún olmo joven (no afectado, por el momento, por dicha enfermedad). Se observa que en las zonas de mayor concentración de olmos muertos, no existen olmos jóvenes vivos mientras que si los hay en la orla y allí donde los chopos eran más frecuentes que los olmos.

La chopera-olmeda es la formación vegetal que ocupa una superficie mayor del Soto y Partinchas. Es también un bosque mixto pero en este caso, los chopos (Populus nigra y Populus alba) son dominantes en detrimento de los olmos que, en su inmensa mayoría, también están muertos ya sea en pie o en el suelo. A medida que los chopos adquieren un mayor dominio del espacio, el sotobosque varía en su organización y recubrimiento; las zarzas y otras espinosas como el majuelo (Crataegus monogyna) no presentan un recubrimiento homogéneo, sino que se agrupan formando rodales muy densos y se alternan en el espacio con áreas con dominio de un estrato herbáceo de gramíneas principalmente, destacando Hordeum murinum.

En la zona más septentrional y oriental es donde hay una mayor presencia de Populus alba $\mathrm{y}$, precisamente, es aquí donde se encuentran algunos olmos adultos todavía vivos; ello indica que la enfermedad se ceba en aquéllas zonas donde los olmos están más próximos entre sí y forman masas casi monoespecíficas. Aunque también hay 
olmos muertos, al no ser dominantes la impresión de desolación y la degradación es mucho menor de manera que se conservan en buena parte las características ambientales previas a la plaga. Por otro lado, el tránsito es mucho más cómodo que en el sector de la olmeda-chopera.

El Soto y Partinchas propiamente dicho contacta por el margen interior (Fig. 3), a través de un pequeño escarpe, con las denominadas formaciones vegetales limítrofes, de escasa superficie pero gran diversidad y valor ecológico. Corresponden a comunidades que han ido colonizando el antiguo tramo del Ebro y que se encuentran en diferentes estadios evolutivos.

En el extremo SO. se ha formado un bosque mixto de ribera que conecta con el río Ebro actual y penetra hacia el interior del Galacho. Es una comunidad valiosa en sí misma pero su localización estratégica le confiere más valor al actuar de barrera de contención natural ante las crecidas del Ebro. Junto a este bosque, pero más alejado del Ebro se ha desarrollado una fresneda de Fraxinus angustifolia. Se trata de un espacio que se inunda de forma periódica alcanzando el agua hasta un metro de altura lo que explica que se encuentre desprovista de sotobosque. Sin embargo, su tránsito no es cómodo por la abundante leña seca existente en el suelo.

En contacto con la fresneda hay un pequeño claro más o menos circular con escaso recubrimiento vegetal siendo fundamentalmente especies herbáceas de carácter nitrófilo al ser una zona de sesteo para el ganado. El aprovechamiento ganadero actual de este espacio se puede constatar por las deyecciones y el pisoteo a lo largo de los pseudosenderos abiertos por el soto.

Más próxima a un lagunazo del interior del Galacho se encuentra una sauceda (Salix alba) que forma una franja de 10 a $15 \mathrm{~m}$. y en la que crecen también algunos tamarices. Al igual que la fresneda tampoco presenta sotobosque dada la elevada densidad de pequeños sauces y las inundaciones periódicas a que se ve sometido este espacio (el nivel de las aguas llega a alcanzar los dos metros). Junto a ella se localiza una pradera de agramen (Paspalum paspalodes) que poco a poco va reduciendo sus dimensiones por el avance del carrizal (Phragmites australis), que está colonizando las láminas de agua del antiguo cauce. Esta franja de sauces contacta con un lagunazo o balseta interior a través de un dique de contención en el que hay sobre todo Populus canadensis, pero también Populus nigra y, ya en contacto con el agua, Tamarix gallica. 


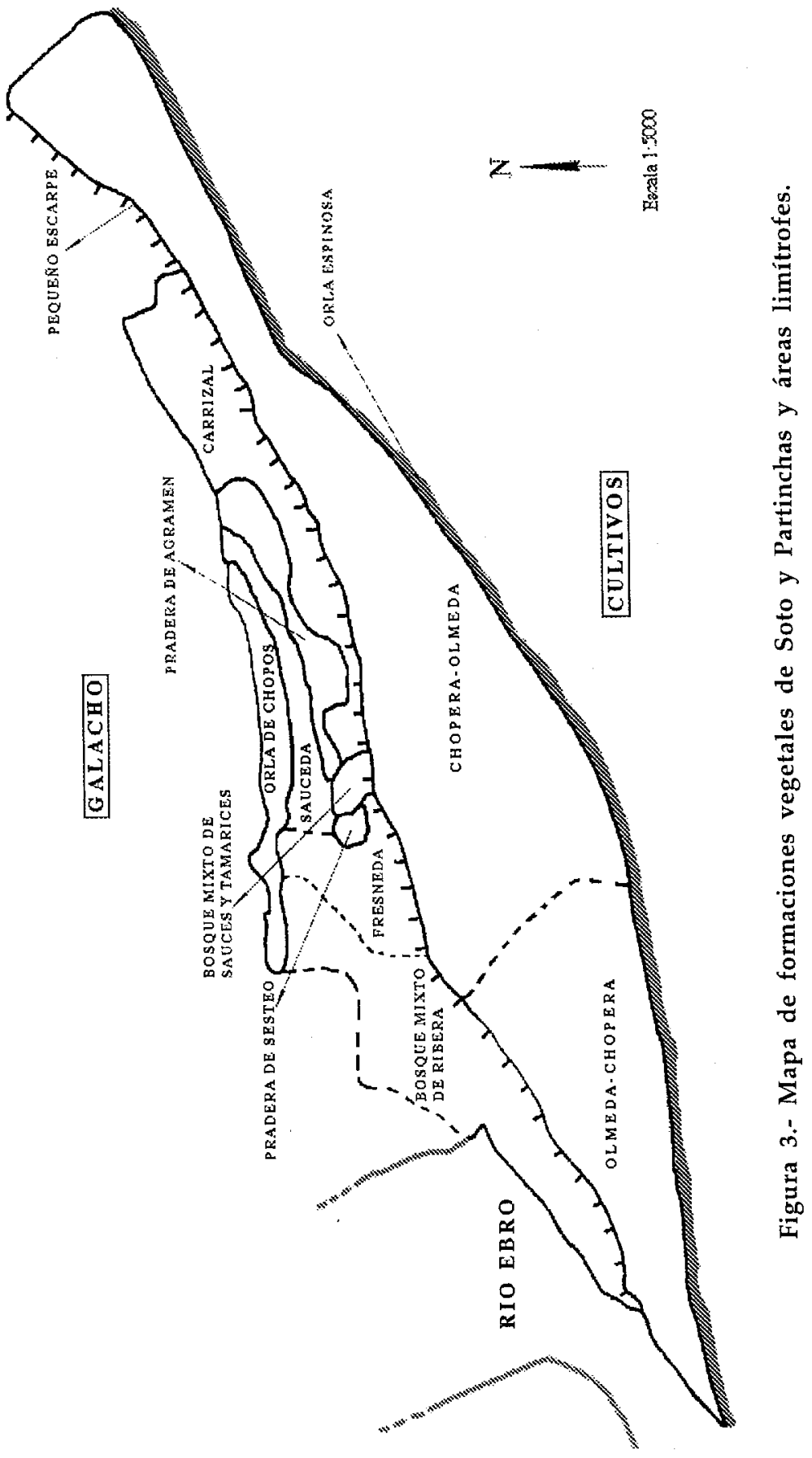




\section{DIAGNOSTICO SOBRE EL ESTADO ACTUAL DE LA VEGETACION Y TENDENCIA EVOLUTIVA PREVISTA}

El diagnóstico del estado actual del Soto y Partinchas se centra en dos aspectos: su estado fitosanitario y limpieza, y su tendencia evolutiva. En cuanto al primer punto, es patente que la población de Ulmus minor está seriamente afectada por la grafiosis, estando casi todos los olmos muertos en el sector de la olmeda-chopera, mientras que todavía quedan algunos en buen estado en el sector de la chopera-olmeda $y$, sobre todo ejemplares jóvenes en la orla exterior. Por consiguiente, la plaga se ha cebado de forma más intensa en las zonas de mayor densidad de olmos y en los ejemplares más maduros.

En cuanto al momento concreto en que la plaga de la grafiosis comenzó a afectar de forma importante a esta arboleda, es difícil saberlo con precisión pues no se ha realizado un seguimiento por parte de ningún organismo al ser propiedad particular y no se ha conseguido tampoco esta información de los propietarios. La ortofoto de 1988 (a escala 1:5.000) es un punto de referencia para evaluar su estado en fechas anteriores a la actual pero no excesivamente lejanas; en esta imagen el soto no parece estar afectado todavía, por lo menos de una forma masiva y ello coincide con las referencias a este espacio incluidas en otros estudios en dichas fechas (Universidad de Zaragoza, 1990). Por lo tanto, hay que inclinarse a pensar que la situación de degradación existente en el momento actual se inició después de 1988 y era manifiesta en 1993, fecha en la que se tiene constancia cierta del deterioro del soto estudiado.

Como consecuencia de esta enfermedad el Soto y Partinchas presenta en la actualidad gran número de troncos de olmos muertos, tanto en pie como caídos en el suelo, al igual que una enorme cantidad de ramas secas amontonadas en el suelo. Este hecho supone, por un lado, un riesgo importante para la integridad física de las personas que circulen por estos espacios pues los olmos muertos que todavía están en pie pueden caer en cualquier momento; por otro lado, favorece la expansión de un posible fuego así como de enfermedades; por último, dificulta enormemente el tránsito por el interior del bosque. Todo esto es aplicable principalmente al sector de la olmedachopera pues al ser mejor el estado fitosanitario de la chopera-olmeda, estos problemas no están tan agudizados. La evidencia de los riesgos y problemas que conlleva la presencia de los olmos y ramas secas en estas arboledas aconseja de forma rotunda una actuación que los solucione 0 , al menos, amortigüe. En este sentido serían necesarias labores silvícolas de limpieza de las ramas y troncos muertos caídos en el suelo así como la tala y retirada cuidadosa de los troncos muertos que permanecen en pie.

En cuanto a la dinámica de la vegetación hay que señalar, en primer lugar, que ha sido regresiva de forma clara en los últimos años en el sector de la olmeda-chopera, 
por las razones ya expuestas. Sin embargo, en la chopera-olmeda así como en la orla, la dinámica puede considerarse estable puesto que los olmos afectados no han llegado a interferir de forma importante en la dinámica general de estas formaciones debido a su menor presencia. Desafortunadamente, en nuestro país no hay indicios de que la grafiosis haya sido controlada por lo que puede suponerse que seguirá causando estragos entre esta especie.

Respecto a la tendencia evolutiva general de estas formaciones arbóreas se puede diagnosticar que, probablemente se producirá un cambio en la composición florística pues el Populus alba irá sustituyendo paulatinamente al Ulmus minor. Dejarán por tanto de ser formaciones arbóreas mixtas para ser choperas monoespecíficas, lo que sin duda es una pérdida lamentable de diversidad y riqueza pero no hace peligrar el futuro del bosque, hecho que se considera fundamental. En definitiva, la tendencia puede permanecer estable o incluso ser progresiva respecto a la situación de degradación actual teniendo en cuenta la elevada capacidad de regeneración que caracteriza, en general, a estos espacios y considerando, desde luego, una gestión adecuada. En este sentido, es de destacar la proximidad del bosque mixto de ribera que constituye un banco de semillas que bien puede colaborar en la regeneración de este espacio.

Hay que destacar, por otro lado, el papel que las formaciones arbóreas del Soto y Partinchas ha tenido y tiene en la dinámica geomorfológica del Ebro y en el impacto de las crecidas sobre los cultivos de Juslibol. En efecto, los bosques de olmos y chopos tan próximos a la orilla han jugado un papel estabilizador del cauce del Ebro durante las crecidas disipando la energía de las aguas y favoreciendo la decantación de materiales en suspensión. Al mismo tiempo constituyen una barrera natural de protección para los campos de cultivos adyacentes, puesto que al ser una zona de alta susceptibilidad erosiva, si se eliminase el estrato arbóreo, el meandro iría ampliando su curvatura con la consiguiente reducción de los campos y la posibilidad de que, en una crecida importante, el dique de contención no fuese suficiente y el agua lo desbordase inundando los campos. En consecuencia, no sólo es valioso este espacio por sus características naturalísticas sino también por la función de protección que desempeña frente a grandes avenidas .

Por último, hay que destacar además, la función de protección que el Soto y Partinchas ejerce sobre las valiosas comunidades vegetales limítrofes, correspondientes ya al propio Galacho, y sobre todo el Galacho en general. Protección no sólo desde el punto de vista geomorfológico e hidrológico, por las razones ya expuestas, sino también porque supone una barrera natural que dificulta el acceso inadecuado de la población por este sector al Galacho. 


\section{CRITERIOS DE GESTION Y ALTERNATIVAS DE ACTUACION}

Los principales tipos de criterios u objetivos que pueden utilizarse en la gestión de un espacio de ribera como el Soto y Partinchas son tres: productivistaseconomicistas, conservacionistas y productivistas-conservacionistas. Dependiendo de ellos, las alternativas de actuación serán bien diferentes por lo que se exponen a continuación agrupadas según dichos criterios.

Criterios productivistas-economicistas. Tienen por objeto obtener la máxima rentabilidad económica del recurso natural y, normalmente, a corto plazo. En el caso que nos ocupa, sería, por ejemplo, la gestión de cara a la producción intensiva de madera. Ello implicaría la tala a mata rasa de la arboleda del Soto y Partinchas (para vender la madera tanto de los chopos como de los olmos muertos) y plantar posteriormente chopos de producción (Populus canadensis, por ejemplo) de ciclo de crecimiento rápido. En cuanto los nuevos chopos plantados alcanzasen un desarrollo que hiciese rentable su venta, serían talados y volverían a plantarse otros; en definitiva, una gestión del soto como un cultivo.

Este tipo de criterios y actuaciones son los que defiende la AsociaciónComunidad de la partida de Soto y Partinchas, propietaria actual de la zona de estudio que ha solicitado en varias ocasiones el permiso para la tala de la olmedachopera y chopera-olmeda con objeto de vender la madera y gestionar en el futuro este espacio como un cultivo de chopos. Hasta el momento actual, el permiso les ha sido denegado aludiendo a los posibles riesgos geomorfológicos e hídricos que una tala indiscriminada del soto conllevaría no solo para el propio Galacho de Juslibol sino también para la zona de cultivos. En efecto, estas formaciones arbóreas ejercen una función de barrera natural y de importante freno de cara a las inundaciones que quedaría completamente eliminada tras la tala y durante los primeros años tras cada tala y, en cualquier caso, definitivamente diezmada, pues una chopera de producción, ni siquiera madura, puede ejercer la misma función que las formaciones vegetales actuales.

A los riesgos geomorfológicos e hidrológicos que se provocarían con una gestión basada en criterios exclusivamente productivistas-economicistas, se añade la importante pérdida de un espacio de elevados valores ambientales que complementa de forma idónea el conjunto protegido del Galacho de Juslibol. Pese al mal estado fitosanitario del sector de la olmeda-chopera, el Soto y Partinchas constituye un espacio con valores suficientes para que sean aplicados en él otros criterios que los exclusivamente productivistas-economicistas.

Criterios conservacionistas. Tienen por objeto proteger espacios de elevados valores naturales para conservar en ellos, o incluso incrementar, las características 
ambientales que los hacen valiosos y asegurar, en la medida de lo posible, su pervivencia futura. Ello implica una gestión encaminada exclusivamente a la buena conservación del recurso sin tener en cuenta su posible explotación como un recurso económico. En muchos casos, las actuaciones necesarias para la conservación de estos preciados espacios tienen un coste económico que puede llegar a ser importante y que, sumado a los beneficios que dejan de percibirse por la no explotación económica de dicho espacio, hacen difícil o casi imposible que estas actuaciones sean abordadas por particulares.

La ubicación junto al Río Ebro y el interés intrínseco que tiene el Soto y Partinchas, justifican su inclusión en el Programa MAB-UNESCO: el Hombre y la Biosfera, su protección en el Plan General de Ordenación Urbana de Zaragoza y su control por parte de la Confederación Hidrográfica del Ebro mediante la aplicación de la Ley de aguas. Es patente que la Administración debe estar implicada de una $\mathbf{u}$ otra manera en la gestión adecuada de este valioso espacio, pero no se puede prescindir de los derechos de quien detenta la propiedad de dicho espacio.

Aplicando estos criterios habría que intervenir en la línea de mejorar el estado fitosanitario de estos bosques de ribera. Sería necesario sacar toda la leña seca acumulada en el suelo así como talar todos los olmos muertos y proceder igualmente a su retirada. Todo ello, por supuesto, realizado con cuidado y sin dañar la vegetación colindante lo que requiere unas labores costosas y controladas. Podría ser aconsejable la trituración "in situ" de la leña seca, lo que evitaría los trabajos de transporte (complicados en algunos sectores) y podría incluso enriquecer el suelo. Por otro lado, sería aconsejable un tratamiento sanitario de los olmos aún vivos que puedan ser afectados por la grafiosis con objeto de posibilitar la mayor diversidad florística de estos bosques, no solo actual sino futura.

Para aplicar este tipo de criterios, lo idóneo sería que los terrenos del Soto y Partinchas pudiesen gestionarse de forma coordinada como parte del Espacio Natural Protegido del Galacho de Juslibol. Por ello, sería conveniente profundizar en el tema de la propiedad del Soto y Partinchas $^{2}$ y, si es posible y hay derecho a ello, reclamar esta propiedad para la entidad municipal o para la Confederación Hidrográfica del Ebro (en caso de que le corresponda a esta entidad en aplicación de la Ley de Aguas). Siendo al menos una parte del Soto y Partinchas de la Administración pública podrían llevarse a cabo más fácilmente actuaciones de tipo conservacionista como las indicadas.

Criterios productivistas-conservacionistas. Estos criterios tratan de compatibilizar la obtención de un cierto rendimiento económico derivado de la

2 La propiedad de las parcelas del Soto y Partinchas es algo confusa pudiendo existir conflictos entre los derechos de la denominada "Asociación Comunidad de Partidas del Soto y Partinchas", la entidad municipal y la Confederación Hidrográfica del Ebro. 
explotación del recurso natural con la conservación del mismo. Son criterios en la línea del denominado "ecodesarrollo" o "desarrollo sostenible" en definitiva, de la búsqueda de un equilibrio entre la explotación de la naturaleza y su preservación para el futuro, entre el beneficio socio-económico y el beneficio también social pero ecológico. Son criterios que tienen, en general, más posibilidades de ser aplicados que los estrictamente conservacionistas pues al posibilitar un cierto beneficio económico puede inducirse más fácilmente a su aplicación a propietarios particulares.

En el caso que nos ocupa las medidas de actuación que pueden proponerse son las de realizar igualmente las tareas de mejora fitosanitaria y de limpieza del bosque (saca de madera seca, tala de los olmos muertos aún en pie...) pero permitir una cierta extracción de madera verde mediante una entresaca cuidadosa y controlada. Incluso se podría plantear una colaboración económica de la Administración (ya sea el Ayuntamiento de Zaragoza o la Confederación Hidrográfica del Ebro) en la financiación de las labores silvícolas que es preciso realizar para conservar en buen estado estos bosques de ribera y que puedan cumplir bien su función hidrogeomorfológica y ecológica en general.

Un aprovechamiento turístico-recreativo y/o educativo bien planificado y controlado puede ser otra línea de actuación que confiera una rentabilidad económica suficiente a sus propietarios de manera que llegase a resultar más ventajosa su gestión como zona de interés naturalístico bien conservada que como zona de producción maderera mediante la sucesiva tala y plantación de chopos.

Las propuestas de actuación referidas a la propiedad señaladas en el epígrafe anterior son igualmente aconsejables para la puesta en práctica de medidas basadas en estos criterios combinados. De cualquier forma, lo deseable e imprescindible es la existencia de entendimiento, diálogo y coordinación entre los distintos implicados: propietarios, Administración, usuarios.

\section{BIBLIOGRAFIA}

BRAUN-BLANQUET, J. y BOLOS, O. de (1954): Datos sobre las comunidades terofíticas de las Ilanuras del Ebro medio. Collect. bot., IV: 235-242. Com. SIGMA, 123. Barcelona.

BRAUN-BLANQUET, J. y BOLOS, O. de (1957): Las comunidades vegetales de la depresión del Ebro y su dinamismo. Delegación Medio Ambiente Ayuntamiento de Zaragoza. Zaragoza.

ECAS TECNICOS ASOCIADOS S.A. (1991): Inventario y caracterización de las formaciones de ribera en Aragón. Fase I: río Ebro y tramos finales de sus principales afluentes. D. G. A. Zaragoza. 
FOLCH i GUILLEN, R. (1981): La vegetació dels Països Catalans. Ed. Ketres. Barcelona.

PELLICER CORELLANO, F. y YETANO RUIZ, L.M. (1985): El Galacho de Juslibol: un ejemplo de meandro abandonado" Cuadernos de investigación geográfica, XI, 113-124, Logroño.

REGATO PAJARES, P. (1988): Contribución al estudio de la flora y la vegetación del «Galacho de la Alfranca» en relación con el sistema fluvial. Naturaleza en Aragón 3. D.G.A. Zaragoza.

VV.AA. (Coord. PELLICER, F.): Estudio de recuperación del Galacho de Juslibol. Ayuntamiento de Zaragoza-Universidad de Zaragoza. 1990. 